\title{
Tradução e Adaptação do Pay Attention! - Um Programa de Treinamento dos Processos da Atenção para Crianças
}

\author{
Translation and Adaptation of Pay Attention! - A Children's Attention \\ Process Training Program
}

\author{
Deise Lima Fernandes Barbosa, Mônica Carolina Miranda* \\ \& Orlando Francisco Amodeo Bueno \\ Universidade Federal de São Paulo, São Paulo, SP, Brasil
}

\begin{abstract}
Resumo
Este estudo descreve o processo de tradução, adaptação e análise de aplicabilidade clínica do Programa Pay Attention! ao português do Brasil. O processo seguiu as etapas recomendadas internacionalmente para adequação de instrumentos a outros idiomas: tradução/adaptação baseado em normas que preservassem características originais bem como as da cultura; análise por juízes especialistas; estudos-piloto; análise de aplicabilidade clínica em um grupo de 10 crianças com TDAH. Os resultados dos estudos-piloto foram analisados qualitativamente na fase de tradução/adaptação. No grupo clínico, o programa foi aplicado em 20 sessões individuais analisando-se a evolução individual, conforme estabelecido no manual. Os resultados demonstraram que a versão em português do Pay Attention! está adequada, tornando-se um importante instrumento de intervenção nos Transtornos de Déficit de Atenção em crianças.

Palavras-chave: Atenção, reabilitação cognitiva, intervenção, Transtorno do Déficit de Atenção e Hiperatividade.
\end{abstract}

\begin{abstract}
This research report describes the translation, adaptation and analysis of clinical applicability process related to the Pay Attention! Program to Brazilian Portuguese. The process followed all the steps internationally recommended to adequate instruments to other languages: translation/adaptation based on rules that preserve original features and cultural characteristics; analysis by expert judges; pilot studies, and analysis of clinical applicability in a group of 10 children with Attention Deficit and Hyperactivity Disorder (ADHD). Results of pilot studies were qualitatively analyzed in the phase of translation/adaptation. In the clinical group, the program was implemented in 20 individual sessions analyzing the individual evolution, as stated in the manual. Results showed that the Portuguese version of the Pay Attention! Program is appropriate, becoming an important tool for the interventions in attention deficits in children.

Keywords: Attention, cognitive rehabilitation, intervention, Attention Deficit and Hyperactivity Disorder.
\end{abstract}

A atenção é um dos sistemas mais importantes e complexos da cognição, do qual dependem outras tantas funções essenciais para a adaptação do organismo ao ambiente, como a memória e a aprendizagem. Devido a essa complexidade o sistema atencional tem sido amplamente estudado em modelos clínicos e experimentais (Driver, 2001; Hampshire, Chamberlain, Monti, Duncan, \& Owen, 2010; Nahas \& Xavier, 2004)

Sohlberg e Mateer (1987) propuseram um dos modelos clínicos da atenção. Em seu "Modelo Clínico Atencional”,

\footnotetext{
* Endereço para correspondência: Rua Embaú, 54, Vila Clementino, São Paulo, SP, Brasil 04039-060. E-mail: mirandambr@yahoo.com.br

Apoio Financeiro: Fundação de Amparo à Pesquisa do Estado de São Paulo (FAPESP); Associação Fundo de Incentivo à Pesquisa (AFIP).
}

o sistema é dividido em cinco níveis de processamento de complexidade crescente: (a) atenção focalizada; (b) atenção sustentada; (c) atenção seletiva, que incorpora o conceito "resistência à distração"; (d) atenção alternada; (e) atenção dividida.

Para outros autores, a atenção também se encontra sob o conceito das Funções Executivas (FEs). Segundo as teorias neuropsicológicas, uma disfunção executiva interfere na capacidade de controlar o comportamento, resultando em problemas de impulsividade e desatenção (Brocki \& Bohlin, 2006).

Os déficits de atenção e FEs estão relacionados a uma variedade de condições clínicas incluindo trauma cerebral, anóxia/hipóxia, distúrbios globais e invasivos do desenvolvimento, exposição pré-natal a toxinas (como drogas e álcool) e distúrbios de aprendizagem. A maioria das crian- 
ças com déficit de atenção é diagnosticada com Transtorno do Déficit de Atenção e Hiperatividade (TDAH), sendo esse um problema de saúde mental bastante frequente em crianças, adolescentes e adultos em todo o mundo. Dados mundiais apontam para uma incidência de 6 a $9 \%$ de crianças e adolescentes e 3 a $5 \%$ de adultos com o TDAH (Dopheide \& Pliszka, 2009).

A reabilitação neuropsicológica ou cognitiva para o tratamento de déficits atencionais tem recebido destaque na literatura internacional. Conforme Sohlberg e Mateer, (2009) a reabilitação cognitiva pode ser direcionada tanto para o manejo de dificuldades acadêmicas como para várias áreas da cognição. Entre as várias modalidades que podem ser utilizadas no processo de reabilitação cognitiva estão os programas de "treinamento" de processos específicos, nos quais os vários componentes cognitivos são vistos como capacidades que podem ser melhoradas através de treinamento (Tamm, Epstein, Peugh, Nakonezny, \& Hughes, 2012) e que segundo Miotto, Serrão, Guerra, Lucia e Scaf (2008), também auxiliam o indivíduo a utilizar efetivamente estratégias compensatórias, generalizando o aprendizado para tarefas da vida diária.

Entre os programas estruturados de treinamento da atenção estão o Attention Process Training (APT; Sohlberg \& Mateer, 2001) e sua versão para a infância, o Pay Attention! - A Children's Attention Process Training Program (Thomson, Seidenstrang, Kerns, Sohlberg, \& Mateer, 2005). O Pay Attention! foi desenvolvido a partir dos princípios do APT para uso em crianças de 4 a 10 anos de idade, com o objetivo de intervir nas dificuldades de atenção sustentada, seletiva, alternada e dividida. Para Tamm et al. (2010) o treinamento da atenção se concentra no treinamento de habilidades atencionais centrais usadas em muitas tarefas (melhorando a eficiência da rede que permite a realização de uma tarefa específica). Por isso, um desempenho melhorado em várias tarefas é esperado, ou seja, a transferência do treinamento.

A eficácia do Pay Attention! e do APT foi analisada em quadros de TDAH e em outras condições clínicas. Em um estudo com crianças sobreviventes de câncer tratadas por quimioterapia, no qual foi utilizado o APT, houve melhora estatisticamente significativa da atenção em todas as três medidas usadas no estudo (Butler \& Copeland, 2002).

No estudo de Chenault, Thompson, Abbot e Berninger (2006), o Pay Attention! foi utilizado em um programa de intervenção com 20 crianças que preencheram critérios para Dislexia. Elas foram aleatoriamente divididas em dois grupos: de tratamento (treinamento da atenção) ou grupo controle (treinamento de fluência em leitura). Os resultados indicaram que não houve transferência direta do treinamento da atenção para as redações escolares, porém houve melhora mais rápida quando o treinamento da atenção foi realizado antes do treino de leitura.

Já em crianças com TDAH, alguns estudos com o Pay Attention! mostraram que houve significativas melhoras em uma série de medidas não-treinadas de atenção e eficiência acadêmica (Kerns, Eso, \& Thompson, 1999); alterações significativas em medidas de atenção visual e auditiva, pré e pós-treino (Semrud-Clikeman, Harrington, Clinton, Connor, \& Sylvester, 1998; Semrud-Clikeman et al., 1999); melhora dos sintomas de TDAH e das FEs relatadas por pais e terapeutas, bem como melhora de desempenho nos testes de raciocínio, flexibilidade cognitiva e memória operacional (Tamm et al., 2010); efeitos significativos da intervenção sobre os sintomas de TDAH (segundo os pais e profissionais clínicos), sobre a capacidade de se concentrar (auto-relato da criança), sobre o funcionamento executivo (relato dos pais), além de melhora em testes neuropsicológicos relacionados à capacidade de planejamento estratégico (Tamm et al., 2012).

De acordo com Hambleton e Patsula (1999), existem algumas razões, citadas na literatura, para que instrumentos existentes sejam traduzidos/adaptados:

1. Adaptar um instrumento é consideravelmente mais barato e mais rápido do que produzir um novo instrumento em uma segunda língua;

2. Pode haver falta de recursos necessários para desenvolver um novo instrumento em uma segunda língua, com qualidade adequada;

3. Há um sentimento de segurança que está associada a um instrumento adaptado mais do que em um recém-desenvolvido, especialmente quando o inicial é bem conhecido (e eficaz), e

4. A equidade muitas vezes resulta da presença de múltiplas versões de um instrumento.

Devido à sua comprovada eficácia em estudos anteriores, Adamson, O'Toole e Petersen (2012), descreveram o uso o Pay Attention! em um Programa de Reabilitação Cognitiva (CRP) abreviado enquanto um procedimento de baixo custo e curto período de aplicação, o que facilitaria as aprovações e pagamento por parte de instituições e seguros de saúde

Dessa forma, os resultados dos estudos internacionais com o Pay Attention!, assim como a necessidade crescente de novas tecnologias em reabilitação em âmbito nacional justificam a realização da adaptação transcultural para o português do Brasil do Pay Attention! - A Children's Attention Process Training Program, objeto do presente estudo.

\section{Método}

O processo de tradução e adaptação foi dividido em duas etapas consecutivas. A primeira etapa foi dividida em três fases: (a) tradução e adaptação do manual, tarefas e estímulos; (b) análise da adequação da tradução e adaptação por juízes especialistas bilíngues; (c) estudos-piloto com crianças sem distúrbios e com diagnóstico de TDAH. A segunda etapa compreendeu a análise de aplicabilidade do instrumento em um grupo de 10 crianças com diagnóstico de TDAH.

\section{Etapa 1 - Tradução e Adaptação}

Descrição das Tarefas. (a) Arranjo de cartões: nos níveis de atenção sustentada, seletiva, alternada e dividida esta tarefa exige que a criança separe os cartões em "pilhas" ou "montes" de acordo com os critérios que aumentam em 
Barbosa, D. L. F., Miranda, M. C. \& Bueno, O. F. A. (2014). Tradução e Adaptação do Pay Attention! - Um Programa de Treinamento dos Processos da Atenção para Crianças.

nível de dificuldade. Exemplo: "Vamos ver o quão rápido você pode separar os cartões em ou 'montes' por cor de cabelo". (b) Busca na casa: nos níveis de atenção sustentada, seletiva, alternada e dividida esta tarefa exige que a criança realize busca visual ativa e cancele características-alvo nos estímulos das casas com uma caneta. Os critérios do cancelamento aumentam em nível de dificuldade pelo número e complexidade das características. Exemplo: "Vamos ver o quão rápido você pode encontrar todas as coisas penduradas nas paredes desta casa. Quando você encontrar uma, faça uma marca sobre ela". (c) Resposta rápida: nos níveis de atenção sustentada e seletiva esta tarefa exige que a criança responda aos estímulos-alvo pressionando uma campainha. As respostas aumentam em nível de dificuldade pelo número e complexidade das características. Exemplo: "Eu vou mostrar para você estes cartões, um de cada vez. Toda vez que você vir um membro da família preta aperte a campainha". (d) Atenção auditiva: nos níveis de atenção sustentada, seletiva, alternada e dividida esta tarefa exige que a criança responda aos estímulos-alvo pressionando uma campainha. As respostas aos critérios aumentam em nível de dificuldade pela complexidade dos estímulos, bem como aumentando-se a velocidade com que a criança tem que responder. Exemplo: "Eu vou dizer os nomes de várias cores diferentes, assim: 'vermelho, azul, amarelo, laranja, verde'. Toda vez que eu disser 'vermelho', aperte a campainha".

As tarefas de Atenção Sustentada exigem que a criança mantenha a atenção por certo período de tempo. As tarefas de Atenção Seletiva demandam capacidades de manutenção da atenção, assim como seletividade dos estímulos-alvo dentre distratores. As tarefas de Atenção Alternada incluem traço de alternância entre dois estímulos-alvo. Já no bloco de Atenção Dividida exige-se execução de duas tarefas ao mesmo tempo, sendo necessário que uma delas já tenha sido exaustivamente treinada e automatizada.

Fase 1: Manual e Instruções. O programa Pay Attention! é composto pelos blocos de tarefas ilustrados na Figura 1. Pode-se verificar o número de tarefas por bloco, assim como por nível de processamento. Estas são organizadas em níveis de dificuldade crescente.

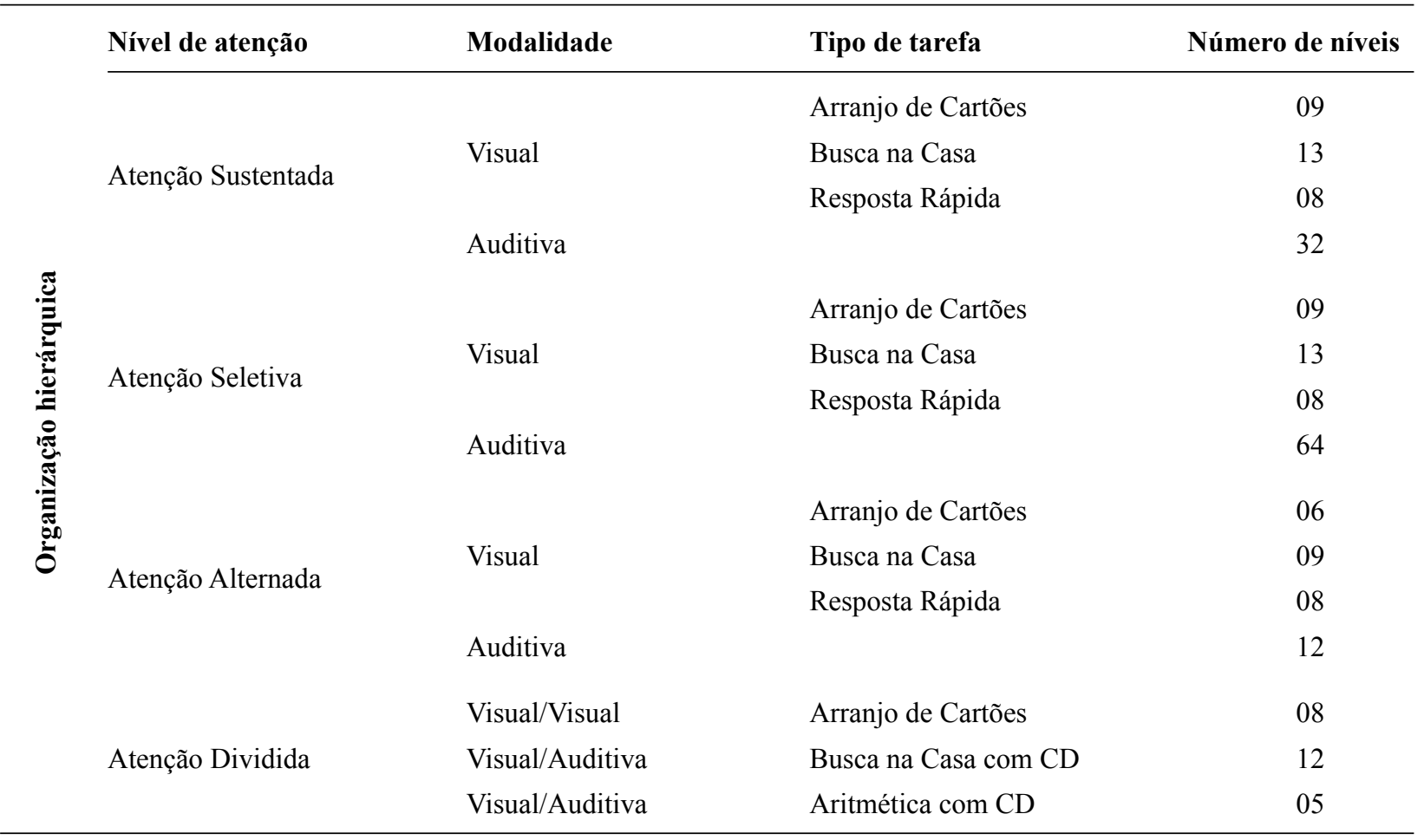

Figura 1. Organização das tarefas do Programa Pay Attention!

$\mathrm{O}$ processo de adaptação iniciou-se com a tradução do manual completo. Este é composto por 52 páginas incluindo o conteúdo das tarefas gravadas em $\mathrm{CD}$, nos Apêndices A, B e C. Após essa etapa ocorreu o processo de adaptação de acordo com o tipo de tarefa.

1. Estímulos visuais: O material das tarefas visuais do Pay Attention! é composto por dois conjuntos de 24 cartões que representam membros de famílias e três figuras de casas (residências das famílias). Os cartões apresentam pessoas de diversas características étnico-culturais e os objetos da casa também fazem parte do cotidiano brasileiro. Sendo assim, os estímulos originais foram mantidos. Houve necessidade apenas de adaptar os estímulos em que havia nomes próprios: "Katy" e "Ryan", que foram adaptados a nomes próprios em português do Brasil ("Carla" e "Rafael").

2. Estímulos auditivo-verbais: o Pay Attention! é composto por 108 tarefas auditivo-verbais. Estas tarefas 
estão gravadas em $4 \mathrm{CDs}$, mais $1 \mathrm{CD}$ de estímulos distratores. Cada tarefa auditiva foi adaptada à língua portuguesa do Brasil, a partir dos critérios de desenvolvimento do instrumento original. Em muitas tarefas foi possível a tradução direta, mas em outras tarefas foi realizada substituição para palavras mais adequadas ao idioma português. Esse processo foi baseado nos seguintes critérios: (a) Frequência na língua portuguesa do Brasil: foram utilizadas as listas de palavras de alta e média freqüência em crianças brasileiras, estudo desenvolvido por Pinheiro (1996). (b) Semelhante estrutura silábica: 16 tarefas no instrumento original continham somente palavras de no máximo duas sílabas, enquanto que nas demais não havia regra de estrutura. Para as tarefas em que havia a exigência de palavras curtas foi feita a adaptação, seguindo a característica original. Também foi considerado como critério o tempo de emissão oral, que deveria ser semelhante ao original. (c) Mesmo som inicial: quando possível, foram escolhidas palavras com o mesmo som inicial das palavras (estímulos) contidas no instrumento original.

Fase 2 - Análise da Tradução e Adaptação por Juizes Especialistas. O comitê de juízes foi formado por um grupo de pesquisadores da Universidade Federal de São Paulo (UNIFESP; neuropsicólogos, fonoaudiólogos, psicólogos cognitivos, entre outros), com amplo conhecimento, assim, em neuropsicologia e áreas afins, além de conhecimento em tradução e adaptação de instrumento, e com fluência no idioma do instrumento original, ou seja o inglês. Os profissionais julgaram a adequação da adaptação de cada um dos estímulos. A partir da avaliação e discussão dos pesquisadores com o comitê de juizes, foi elaborada uma versão atualizada com base nesse julgamento. Essa versão dos estímulos auditivos foi enviada a um estúdio de gravação profissional, assegurando manter as características originais de tempo de apresentação dos estímulos (apresentação lenta com intervalo de 2 segundos e apresentação rápida com intervalo de 1 segundo).

Fase 3 -Estudos-Piloto. Estudo-piloto 1: $\mathrm{O} 1^{\circ}$ estudo-piloto foi realizado com o objetivo de analisar as primeiras modificações do instrumento. Realizou-se a aplicação das primeiras e últimas tarefas dos blocos de treinamento do Pay Attention! em crianças com diagnóstico de TDAH atendidas no ambulatório do Núcleo de Atendimento Neuropsicológico Infantil (NANI). Como as tarefas em cada bloco e modalidade apresentam as mesmas instruções (exceto estímulo/critério-alvo), foram selecionadas as consideradas mais fáceis e as mais difíceis, com o objetivo de verificar a compreensão das instruções, assim como dos estímulos. Os participantes foram dois meninos, (8 e 12 anos de idade), que já estavam em acompanhamento medicamentoso no ambulatório. Foram realizadas cinco sessões individuais, semanais, com cada um deles, totalizando 10 sessões de 50 (cinquenta) minutos cada.
O desempenho, dúvidas e comentários das crianças durante a execução das tarefas foram registrados para darem base à análise qualitativa dos dados e modificação do manual e/ou estímulos.

Estudo-piloto 2: foi realizado um $2^{\circ}$ estudo-piloto com crianças sem distúrbios do desenvolvimento com o objetivo de analisar de forma mais ampla a adequação dos estímulos, bem como a compreensão das instruções em português. A seleção dos participantes e a aplicação das tarefas foram realizadas em duas escolas públicas da região central da cidade de São Paulo.

A amostra foi composta por 18 crianças, com idades entre 04 e 12 anos, sendo uma de cada idade e sexo. Para a seleção dos participantes foram aplicados dois testes de rastreio, a Escala Conners Abreviada para professores (Brito, 1999) e um questionário breve sobre as condições de desenvolvimento da criança, respondido pelos pais/ responsáveis. Foram excluídas aquelas que apresentaram indício de hiperatividade, outros distúrbios do neurodesenvolvimento e uso de medicação.

Nas crianças entre 4 e 6 anos foram aplicadas todas as tarefas de pré-treino por serem indicadas para crianças pequenas ou com graves comprometimentos cognitivos, em 1 sessão de cerca de 40 minutos. As tarefas de pré-treino são tarefas mais simples que as do bloco de treinamento. Exemplo: "Eu vou dizer os nomes de várias coisas diferentes. Quando eu disser alguma coisa que é grande, você vai dizer 'sim'. OK? Vamos lá!"

Com as crianças entre 07 e 12 anos foram utilizadas 2 sessões, de cerca de 50 minutos cada. O critério de seleção de tarefas foi o mesmo utilizado no estudo-piloto 1 . O desempenho, dúvidas e comentários das crianças durante a execução foram registrados para darem base à análise qualitativa dos dados e modificação do manual.

Todos os procedimentos dos estudos foram aprovados pelo Comitê de Ética da UNIFESP e os pais/reponsáveis assinaram Termo de Consentimento Livre e Esclarecido.

\section{Etapa 2 - Análise de Aplicabilidade Clínica do Instrumento}

Participantes. Participaram dessa fase 10 crianças com diagnóstico de TDAH (07 meninos e 03 meninas) com idade média de 9,9 anos (DP 2,5), os quais constituiriam o grupo-alvo para aplicação do Pay Attention!. As crianças foram recrutadas do Ambulatório do Núcleo de Atendimento Neuropsicológico Infantil (NANI) no qual foi desenvolvida esta pesquisa. O diagnóstico foi realizado por uma equipe interdisciplinar que compreende avaliação neurológica, psiquiátrica e neuropsicológica através dos seguintes instrumentos: (a) Conners' Continuous Performance Test (CCPT; Conners, 2002); (b) Escala Wechsler de Inteligência para crianças (WISC-III) - QI estimado; (c) Automated Working Memory Assessment reduzida (Bateria AWMA; Alloway, 2007); Breve Inventário das Funções Executivas (BRIEF; Carim, Miranda, \& Bueno, 2012); Child Behavior Checklist (CBCL; Achenbach, 1991); Escala de Avaliação do Comportamento Infantil (EACI-P; Brito, 1999). 
Barbosa, D. L. F., Miranda, M. C. \& Bueno, O. F. A. (2014). Tradução e Adaptação do Pay Attention! - Um Programa de Treinamento dos Processos da Atenção para Crianças.

Foram excluídas crianças com idade inferior a 6 anos e superior a 14 anos; deficiência intelectual, sinais de traumatismo crânio-encefálico, crises convulsivas, transtorno global do desenvolvimento, comorbidades graves que necessitavam de politerapia.

Procedimentos. O processo seguiu a orientação do programa Pay Attention! para a organização hierárquica dos níveis de atenção, a saber: das tarefas de atenção sustentada, seguindo para atenção seletiva, alternada e dividida, bem como o aumento crescente de dificuldade das tarefas. Foram seguidas as normas de interrupção e repetição de tarefas na tomada de linha de base e treinamento contidas no instrumento original. O programa de treino iniciou-se com a tomada de linha de base do desempenho em cada nível de atenção. Aplicaram-se as primeiras sub-tarefas da

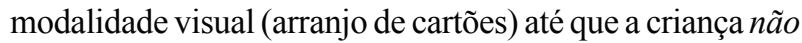
obtivesse $90 \%$ de acertos. A sequência era interrompida e o mesmo processo era feito com a modalidade auditiva. $\mathrm{O}$ mesmo procedimento foi executado nos níveis de atenção sustentada, seletiva e alternada. Para atenção dividida, somente o bloco de modalidade visual/visual (arranjo de cartões) foi aplicado com o mesmo critério de interrupção, pois no nível de atenção dividida uma das tarefas deveria estar automatizada, segundo instrução do instrumento original. O número de sessões válidas utilizadas para esta fase variou entre 03 e 06, dependendo do desempenho individual.

Após a tomada de linha de base, as crianças iniciavam o treinamento de cada nível de atenção a partir da tarefa em que a tomada de linha de base havia sido interrompida. Cada tarefa era aplicada e repetida (se necessário) até que a criança obtivesse $90 \%$ de aproveitamento. Por depender do desempenho individual, o nível alcançado por cada criança diferiu. Foram utilizadas entre 10 e 15 sessões válidas para o treinamento. Um registro detalhado e individual do desempenho quantitativo e qualitativo, tarefa a tarefa, foi realizado.
Análise dos Dados. Estudos-pilotos: com base nos registros dos estudos-pilotos, executou-se um levantamento qualitativo das dúvidas e dificuldades apresentadas na execução das tarefas e a partir disto foram feitas as modificações necessárias para garantir melhor compreensão das instruções.

Aplicabilidade clínica: o desempenho de cada um dos participantes com TDAH foi analisado individual e qualitativamente, comparando-se o tempo de execução (somente nas tarefas de modalidade visual) e número de erros (modalidades visual e auditiva) no nível alcançado na linha de base e reaplicado na fase de treino em cada um dos níveis de atenção.

\section{Resultados}

\section{Tradução, Adaptação e Análise da Adequação}

A análise de adequação da tradução e da adaptação dos estímulos auditivos feita pelos juízes especialistas mostrou necessidade de poucas alterações. Houve sugestões para a adaptação de dois pares de estímulos de tarefas, nas quais a criança responde quando ouve o nome de um animal e depois o movimento que ele faz. O movimento-alvo "hop" foi inicialmente traduzido como PULA, porém os especialistas julgaram que usar o sinônimo SALTA possibilitaria melhor compreensão; o grupo de estímulos-alvo "monkey/swing" traduzido gerou MACACO/BALANÇA. Os juízes sugeriram trocar por PEIXE/NADA. As sugestões feitas pelos especialistas foram posteriormente aceitas e incorporadas à primeira versão do manual em português.

A análise qualitativa do estudo-piloto 1 (crianças com TDAH) mostrou necessidade de modificações nas instruções de algumas tarefas. As modificações foram baseadas nas dúvidas e erros devidos à não compreensão que as crianças apresentaram durante a aplicação das tarefas selecionadas. A Tabela 1 apresenta as modificações realizadas.

Tabela 1

Alterações Realizadas nos Estímulos do Pay Attention! a partir do Estudo-Piloto 1

$1^{\mathrm{a}}$ versão em Português

No manual o paciente era sempre chamado de estudante

Nas tarefas em que a criança tem que separar cartões de acordo com um critério: Chapéu

Moreno (relacionado à cor de cabelo)

Nas tarefas em que a criança tem que marcar características-alvo nos estímulos das casas não havia expresso o número de alvos a serem cancelados.

Nas tarefas em que a criança deve apertar o botão toda vez que o examinador apresenta uma carta com determinada característica: Óculos seguidos por chapéu
Motivo da alteração

Versão Final Adaptada

Por não se tratar de uma lição ou de uma aula, mas Substituída a palavra sim de um programa de intervenção em reabilitação. estudante por criança

Percebeu-se que as crianças separavam somente os cartões em que as pessoas usavam chapéu e deixavam de incluir as pessoas usando boné, não atendendo ao critério original.

As crianças separavam somente cartões em que as pessoas tinham a pele escura.

Pessoa de cabelo escuro

O examinador precisava desta informação para considerar o número de acertos e erros cometidos.

Inserido o número de alvos para cada característica

Não ficava claro para a criança que ela deveria olhar Pessoa com óculos as características encontradas nas pessoas. seguida por pessoa com Chapéu/boné 
Neste estudo também notou-se uma dificuldade na aplicação das tarefas de atenção alternada da modalidade auditiva. Por isto, criou-se um CD não existente na versão original do treino - que utilizava as faixas do CD 1 , precedidas de uma instrução apresentada oralmente pelo treinador. A criança ouvia duas instruções diferentes para a mesma tarefa (uma do CD e outra do treinador) e isto gerava dúvidas na execução. Assim, foi gravado um CD somente com os estímulos, sem instrução alguma, e o treinador oferece as instruções oralmente.

No estudo-piloto 2 a análise das dúvidas e comentários das crianças durante a execução das tarefas mostrou que não havia necessidade de outras modificações nas instruções. As crianças compreendiam o que lhes era solicitado e executavam as tarefas da maneira correta, cometendo erros de ordem atencional, somente (erros de omissão e/ ou erros de ação). Portanto, este estudo piloto não gerou uma nova versão do manual.
Teste de Aplicabilidade Clínica do Programa Adaptado

As Tabelas 2 e 3 mostram a evolução de cada uma das crianças com TDAH ao longo do programa de treinamento, comparando-se o tempo de execução em segundos (somente nas tarefas de modalidade visual) e número de erros (modalidades visual e auditiva) no nível alcançado na linha de base, que foi reaplicado na fase de treino. Nota-se que, em alguns casos, a criança alcançou o nível máximo na linha de base em dada modalidade e nível de atenção, ou ainda não alcançou os níveis avançados dentro do período que compreendeu as 20 sessões. Vale ressaltar que, por tratar-se de um processo de intervenção, o Pay Attention! não gera escores de desempenho e não há previsão de como as crianças devam evoluir dentro do programa. Assim, não é possível avaliar se os resultados obtidos por cada uma delas são os esperados para idade e sexo, até porque leva-se em consideração as caracteristicas individuais da criança.

Tabela 2

Desempenho da Amostra com TDAH nos Níveis de Atenção Sustentada e Seletiva do Pay Attention!

\begin{tabular}{|c|c|c|c|c|c|c|c|c|c|c|c|}
\hline \multirow[b]{3}{*}{ Casos } & \multirow[b]{3}{*}{ FASE } & \multicolumn{5}{|c|}{ Atenção Sustentada } & \multicolumn{5}{|c|}{ Atenção Seletiva } \\
\hline & & \multicolumn{3}{|c|}{ Visual } & \multicolumn{2}{|c|}{ Auditiva } & \multicolumn{3}{|c|}{ Visual } & \multicolumn{2}{|c|}{ Auditiva } \\
\hline & & Tempo (s) & Erros & Nível & Erros & Nível & Tempo(s) & Erros & Nível & Erros & Nível \\
\hline \multicolumn{12}{|l|}{ Subtipo desatento } \\
\hline \multirow[t]{2}{*}{$1-$ masc, 6 anos } & LB & 70 & 8 & \multirow{2}{*}{5} & 2 & \multirow{2}{*}{5} & 212 & 19 & \multirow{2}{*}{7} & 2 & \multirow{2}{*}{4} \\
\hline & TREINO & 115 & 0 & & 0 & & 161 & 2 & & 1 & \\
\hline \multirow[t]{2}{*}{$2-$ masc, 8 anos } & LB & 96 & 5 & \multirow{2}{*}{6} & 3 & \multirow{2}{*}{6} & 89 & 6 & \multirow{2}{*}{5} & 6 & \multirow{2}{*}{6} \\
\hline & TREINO & 79 & 0 & & 1 & & 62 & 0 & & 1 & \\
\hline \multirow[t]{2}{*}{$3-$ masc, 14 anos } & LB & 47 & 0 & \multirow{2}{*}{9} & 2 & \multirow{2}{*}{10} & 38 & 1 & \multirow{2}{*}{9} & 3 & \multirow{2}{*}{28} \\
\hline & TREINO & -- & & & 1 & & -- & & & 1 & \\
\hline \multicolumn{12}{|l|}{ Subtipo combinado } \\
\hline \multirow[t]{2}{*}{4 - fem, 6 anos } & LB & 205 & 1 & \multirow{2}{*}{9} & 6 & \multirow{2}{*}{2} & 362 & 4 & \multirow{2}{*}{7} & 4 & \multirow{2}{*}{2} \\
\hline & TREINO & 132 & 0 & & 0 & & 414 & 0 & & 1 & \\
\hline \multirow[t]{2}{*}{5 - fem, 8 anos } & LB & 214 & 9 & 7 & 2 & 2 & 148 & 5 & 0 & 1 & 2 \\
\hline & TREINO & 114 & 1 & 1 & 0 & 2 & 83 & 0 & 3 & 0 & 2 \\
\hline 6 - masc, 9 anos & LB & 113 & 5 & 5 & 6 & 2 & 286 & 5 & 7 & 2 & 2 \\
\hline & TREINO & 81 & 1 & & 1 & & 253 & 1 & & 1 & \\
\hline 7 - masc, 9 anos & LB & 90 & 13 & 2 & 3 & ? & 101 & 12 & 5 & 5 & 6 \\
\hline & TREINO & 62 & 3 & 2 & 0 & 2 & 83 & 0 & 3 & 1 & 0 \\
\hline 8 - masc, 10 anos & LB & 68 & 24 & 7 & 3 & 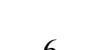 & 73 & 10 & 6 & 2 & 2 \\
\hline & TREINO & 123 & 1 & & 1 & & 81 & 0 & & 0 & \\
\hline 9 - fem, 11 anos & LB & 40 & 5 & 7 & 3 & 8 & 64 & 3 & 9 & 1 & 4 \\
\hline & TREINO & 108 & 0 & 1 & 1 & 8 & -- & & 9 & 0 & 4 \\
\hline 10. masc, 12 anos & LB & 54 & 2 & 0 & 2 & 8 & 59 & 14 & 6 & 4 & 66 \\
\hline & TREINO & -- & & 8 & 0 & 0 & 58 & 0 & 0 & 1 & 00 \\
\hline
\end{tabular}

Nota. LB: linha de base; -- paciente alcançou o nível máximo da linha de base.

A classificação dos subtipos seguiu os critérios do DSM-IV, devido ao período da coleta ser anterior à publicação do DSM 5. 
Barbosa, D. L. F., Miranda, M. C. \& Bueno, O. F. A. (2014). Tradução e Adaptação do Pay Attention! - Um Programa de Treinamento dos Processos da Atenção para Crianças.

Tabela 3

Desempenho da Amostra com TDAH nos Níveis de Atenção Alternada e Dividida do Pay Attention!

\begin{tabular}{|c|c|c|c|c|c|c|c|c|c|}
\hline \multirow[b]{3}{*}{ Casos } & \multirow[b]{3}{*}{ FASE } & \multicolumn{5}{|c|}{ Atenção Alternada } & \multicolumn{3}{|c|}{ Atenção Dividida } \\
\hline & & \multicolumn{3}{|c|}{ Visual } & \multicolumn{2}{|c|}{ Auditiva } & \multicolumn{3}{|c|}{ Visual } \\
\hline & & Tempo (s) & Erros & Nível & Erros & Nível & Tempo(s) & Erros & Nível \\
\hline \multicolumn{10}{|l|}{ Subtipo desatento } \\
\hline \multirow[t]{2}{*}{1 - masc, 6 anos } & LB & 59 & 3 & \multirow{2}{*}{5} & 4 & \multirow{2}{*}{2} & 49 & 8 & \multirow{2}{*}{1} \\
\hline & TREINO & $\ell$ & & & 0 & & e & & \\
\hline \multirow[t]{2}{*}{$2-$ masc, 8 anos } & LB & 73 & 9 & \multirow{2}{*}{6} & 1 & \multirow{2}{*}{1} & 129 & 10 & \multirow{2}{*}{1} \\
\hline & TREINO & 86 & 0 & & 0 & & 96 & 0 & \\
\hline \multirow[t]{2}{*}{3 - masc, 14 anos } & LB & 56 & 2 & \multirow{2}{*}{6} & 4 & \multirow{2}{*}{8} & 73 & 0 & \multirow{2}{*}{8} \\
\hline & TREINO & - & & & 1 & & - & & \\
\hline \multicolumn{10}{|l|}{ Subtipo combinado } \\
\hline \multirow[t]{2}{*}{4 - fem, 6 anos } & LB & 72 & 7 & \multirow{2}{*}{6} & 1 & \multirow{2}{*}{2} & 299 & 9 & \multirow{2}{*}{3} \\
\hline & TREINO & $\ell$ & & & 0 & & $\ell$ & & \\
\hline \multirow[t]{2}{*}{5 - fem, 8 anos } & LB & 122 & 11 & \multirow{2}{*}{6} & 4 & \multirow{2}{*}{2} & 147 & 6 & \multirow{2}{*}{2} \\
\hline & TREINO & $\ell$ & & & -- & & $\ell$ & & \\
\hline \multirow[t]{2}{*}{6 - masc, 9 anos } & LB & 118 & 9 & \multirow{2}{*}{2} & 4 & \multirow{2}{*}{2} & 109 & 5 & 1 \\
\hline & TREINO & 53 & 0 & & 0 & & 97 & 0 & 1 \\
\hline 7 - masc, 9 anos & LB & 89 & 9 & 4 & 1 & 5 & 111 & 6 & 1 \\
\hline & TREINO & 78 & 2 & 7 & 0 & J & 91 & 0 & 1 \\
\hline 8 - masc, 10 anos & LB & 86 & 3 & 3 & 2 & $?$ & 124 & 20 & 3 \\
\hline & TREINO & 49 & 0 & 3 & 0 & 2 & 63 & 1 & 3 \\
\hline 9 - fem, 11 anos & LB & 54 & 5 & 5 & 3 & 6 & 93 & 8 & 3 \\
\hline & TREINO & 44 & 0 & . & 0 & o & 54 & 0 & 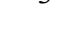 \\
\hline 10 - masc, 12 anos & LB & 43 & 0 & 6 & 2 & 3 & 90 & 6 & 1 \\
\hline & TREINO & - & & 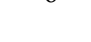 & 0 & 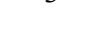 & 66 & 0 & . \\
\hline
\end{tabular}

Nota. LB: linha de base; -- paciente alcançou o nível máximo da linha de base; Ø paciente não alcançou o nível.

A classificação dos subtipos seguiu os critérios do DSM-IV, devido ao período da coleta ser anterior à publicação do DSM 5 .

Descrição do desempenho nos níveis de atenção sustentada e seletiva (Tabela 2): nos casos de subtipo desatento, verificou-se diminuição do tempo de execução de tarefas, aliada à diminuição dos erros, o que pode demonstrar que houve incremento da velocidade e qualidade de processamento dentro do programa. Nos casos de subtipo combinado e hiperativo/impulsivo, verifica-se diminuição do tempo de execução nas crianças mais jovens (6 a 9 anos), enquanto que se observa o oposto no tempo de execução nas crianças mais velhas, também aliado à diminuição dos erros. Estes resultados podem indicar que houve regulação do tempo de execução para obtenção de melhor desempenho

Descrição do desempenho nos níveis de atenção alternada e dividida (Tabela 3): nos níveis de atenção alternada e dividida (que recrutam mais recursos de processamento) nota-se novamente que as crianças mais novas têm mais dificuldades, não alcançando níveis mais elevados de processamento atencional. Já as crianças mais velhas dimi- nuem o tempo de execução e o número de erros. As duas crianças mais velhas conseguiram alcançar níveis máximos nas modalidades visuais já na linha de base.

\section{Discussão}

Este estudo teve como objetivo principal a tradução e adaptação do programa Pay Attention! para o português do Brasil analisando, adicionalmente, a aplicabilidade clínica do programa em um grupo de crianças com diagnóstico de TDAH.

No processo de adaptação foram necessárias substituições somente nos grupos de estímulos auditivos, e essas foram feitas a partir de critérios recomendados pela literatura internacional (Hambleton \& Patsula, 1999), que garantiram a equivalência semântica e cultural com o instrumento original. Para tanto, determinou-se características prévias que foram atendidas para a escolha de palavras substitutas: semelhante estrutura silábica (extensão) e som inicial aos 
dos estímulos originais, considerando a necessidade de que os estímulos atendessem às características peculiares da língua portuguesa do Brasil. A escolha dos estímulos foi baseada em uma pesquisa nacional de frequência de palavras do nosso idioma (Pinheiro, 1996) em crianças de faixas etárias compreendidas pelo Pay Attention! a qual permite, segundo a autora, que se utilizem materiais sobre os quais as crianças tenham maior conhecimento podendo aprimorar a qualidade e confiabilidade.

$\mathrm{Na}$ análise por um comitê de especialistas, foi sugerida a alteração de dois pares de estímulos que se referiam a movimentos de animais. Esse processo garantiu que o significado das expressões ou palavras traduzidas contivessem o mesmo conceito do instrumento original (Beaton, Bombardier, Guillemin, \& Ferraz, 2000; Van de Vijver \& Hambleton, 1996). Essas alterações tornaram os dois grupos de estímulos mais adequados ao cotidiano das crianças brasileiras, possibilitando, além de melhor compreensão, maior identificação com a tarefa, adequando o programa do ponto de vista transcultural.

No que se refere ao processo de tradução, a literatura recomenda, quando se trata de instrumentos de avaliação (como por exemplo, questionários de comportamento), que seja realizada tradução e retro-tradução (Beaton et al., 2000), com o objetivo de evitar significados inesperados nos itens que possam interferir nos dados da normatização. Porém, no caso do Pay Attention!, que se trata de treino cognitivo, em que o enfoque está na instrução da tarefa, a adequação da tradução foi analisada através dos estudos-piloto e posteriormente aos ajustes iniciais, o teste de aplicabilidade clínica.

Tanto o primeiro estudo-piloto como o teste de aplicabilidade foram realizados com crianças com TDAH, processos considerados como "pré-testes" na literatura (Beaton et al., 2000). Esse autor recomenda que o instrumento seja aplicado em indivíduos pertencentes ao grupo-alvo. No primeiro estudo-piloto foram aplicadas tarefas do programa em duas crianças com déficit atencional e foi possível verificar que mesmo atendidas às etapas anteriores sugeridas, somente o contato dos pacientes com o instrumento forneceria a análise adequada da adaptação das instruções assim como das tarefas, visto que algumas alterações nas instruções foram necessárias para possibilitar maior compreensão.

O estudo piloto 2 foi necessário para que se verificasse se crianças sem distúrbios também teriam compreensão das instruções, ou seja, as instruções compreendidas por crianças com TDAH deveriam ser compreendidas por crianças sem o quadro, eliminando um possível viés de amostra clínica. A análise qualitativa deste estudo-piloto confirmou a adequação da nova versão traduzida e adaptada, pois verificou-se que não houve necessidade de novas modificações nas instruções ou nos estímulos, permitindo que essa se constituísse versão final para a análise da aplicabilidade do Pay Attention! em processos de intervenção clínica.
Quanto a essa fase, as recomendações internacionais em instrumentos de avaliação/diagnóstico estão voltadas à análise de propriedades psicométricas originais. O Pay Attention! não gera escores de desempenho, mas sim um registro de acertos, erros e observações qualitativas totalmente individuais que servem para nortear a aplicação do treino cognitivo. Estes registros ajudam o profissional clínico a dar ênfase nas maiores dificuldades e não geram dados comparáveis com amostras normativas. Assim, considerou-se relevante a análise de aplicabilidade utilizando o programa em um grupo clínico.

Faz-se necessário salientar que esta fase do estudo não pretendeu analisar a eficácia do programa para o tratamento de déficits atencionais ou mesmo do TDAH, mas sim analisar a viabilidade de aplicação do programa Pay Attention! traduzido e adaptado para o português em crianças com esta problemática.

Sendo assim, a análise qualitativa da evolução individual dos participantes mostrou que em todos os casos houve compreensão das instruções, dos estímulos e uma evolução nos padrões de tempo de execução e número de erros cometidos quando comparados à linha de base e fase de treinamento.

Tanto no grupo de crianças de subtipo desatento como no de crianças do subtipo combinado, verificou-se que houve uma auto-modulação do tempo de execução que, como consequência, levou à diminuição do número de erros cometidos, sendo esta uma das metas do programa original. Isto mostra que o instrumento encontra-se adequadamente adaptado. Foi também possível observar diferenças no desempenho individual relacionados à idade e consequentemente ao nível de maturação, revelando que a tradução/ adaptação pode ser sensível a estas características.

Porém, para verificação de eficácia no tratamento de déficits atencionais, serão necessários estudos com tamanho amostral adequado e análise comparativa entre os subtipos de TDAH. Sendo assim, conclui-se que: (a) esta pesquisa disponibiliza para uso em crianças brasileiras o programa Pay Attention! A Children's Attention Process Training Program. (b) O processo de tradução e adaptação seguiu os procedimentos recomendados pela literatura, seguindo critérios rigorosos com o objetivo de garantir a adaptação transcultural do Pay Attention! bem como a equivalência com o instrumento original. (c) Os resultados da análise com o grupo clínico mostraram que a versão em português do manual, instruções e estímulos são aplicáveis às crianças brasileiras. (d) Esta versão adaptada pode ser considerada a versão final do instrumento para uso por profissionais da saúde em nosso meio, no tratamento de crianças com déficits atencionais.

\section{Referências}

Achenbach, T. (1991). Manual for the Child Behavior Checklist/4-18 and 1991 profile. Burlington, VT: University of Vermont. 
Barbosa, D. L. F., Miranda, M. C. \& Bueno, O. F. A. (2014). Tradução e Adaptação do Pay Attention! - Um Programa de Treinamento dos Processos da Atenção para Crianças.

Alloway, T. P. (2007). Automated working memory assessment: Manual. London: Pearson.

Adamson, A. M., O’Toole, K., \& Petersen, C. (2012). Establishing a pediatric cognitive rehabilitation program: Insurance issues and clinical rationale. The Clinical Neuropsychologist, 26, 59-73. doi:10.1080/13854046.2011.639313

Beaton, D. E., Bombardier, C., Guillemin, F., \& Ferraz, M. B. (2000). Guidelines for the process of cross-cultural adaptation of Self-Report Measures. Spine, 25(24), 3186-3191. doi:10.1097/00007632-200012150-00014

Brito, G. N. O. (1999). EACI-P - Escala de Avaliação do Comportamento Infantil para o Professor: Manual. São Paulo, SP: Entreletras.

Brocki, K. C., \& Bohlin, G. (2006). Developmental change in the relation between executive functions and symptoms of $\mathrm{ADHD}$ and co-occurring behavior problems. Infant and Child Development, 15, 19-40. doi:10.1002/icd.413

Butler, R. W., \& Copeland, D. R. (2002). Attentional processes and their remediation in children treated for cancer: A literature review and the development of a therapeutic approach. Journal of the International Neuropsychological Society, 8(1), 115-124. doi:10.1017/S1355617701020112

Carim, D. B., Miranda, M. C., \& Bueno, O. F. A. (2012). Tradução e adaptação para o português do Behavior Rating Inventory of Executive Function - BRIEF. Psicologia: Reflexão \& Critica, 25(4), 653-661. doi:10.1590/S010279722012000400004

Chenault, B., Thompson, J., Abbott, R. D., \& Berninger, V. W. (2006). Effects of prior attention training on child dyslexic's response to composition instruction. Developmental Neuropsychology, 29(1), 243-260. doi:10.1207/ s15326942dn2901 12

Conners, C. K. (2002). Conner's Continuous Performance Test. Toronto, Canada: Multi-Health System.

Dopheide, J. A., \& Pliszka, S. R. (2009). Attention deficit hyperactivity disorder: An update. Pharmacotherapy, 29, 656-679. doi:10.1592/phco.29.6.656

Driver, J. (2001). A selective review of selective attention research from the past century. British Journal of Psychology, 92, 53-78. doi:10.1348/000712601162103

Hambleton, R. K., \& Patsula, L. (1999). Increasing the validity of adapted tests: Myths to be avoided and guidelines for improving test adaptation practices. Journal of Applied Testing Technology, 1, 1-12.

Hampshire, A., Chamberlain, S. R., Monti, M. M., Duncan, J., \& Owen, A. M. (2010). The role of the right inferior frontal gyrus: Inhibition and attentional control. Neuroimage, 50(3-3), 1313-1319. doi:10.1016/j.neuroimage.2009.12.109

Kerns, K., Eso, K., \& Thompson, J. B. (1999). Investigation of a direct intervention for improving attention in young children with ADHD. Developmental Neuropsychology, 16(2), 273295. doi:10.1207/S15326942DN1602 9

Miotto, E. C., Serrão, V. T., Guerra, G. B., Lucia, M. C. S., \& Scaf, M. (2008). Cognitive rehabilitation and mild cognitive impairment: A review of the literature. Dementia \& Neuropsychologia Journal, 2(2), 139-145.

Nahas, T. R., \& Xavier, G. F. (2004). Neurobiologia da atenção visual. In V. M. Andrade, F. H. Santos, \& O. F. A. Bueno, Neuropsicologia hoje (pp. 101-124). São Paulo, SP: Artes Médicas.

Pinheiro, A. M. V. (1996). Contagem de frequência de ocorrência de palavras expostas a crianças na faixa pré-escolar e séries iniciais do $1^{\circ}$. grau. São Paulo, SP: Associação Brasileira de Dislexia.
Semrud-Clikeman, M., Harrington, K., Clinton, A., Connor, R. T., \& Sylvester, L. (1998). Attention functioning in two groups o ADHD children with and without attention training interventions. Paper presented at the $26^{\text {th }}$ Annual International Neuropsychological Society Conference, Honolulu, Hawaii.

Semrud-Clikeman, M., Nielsen, K. H., Clinton, A., Sylvester, L., Parle, N., \& Connor, R. T. (1999). An intervention approach for children with teacher and parent identified attentional difficulties. Journal of Learning Disabilities, 32(6), 581-590. doi:10.1177/002221949903200609

Sohlberg, M. M., \& Mateer, C. A. (1987). Effectiveness of an attention training program. Journal of Clinical and Experimental Neuropsychology, 9, 117-130. doi:10.1080/01688638708405352

Sohlberg, M. M., \& Mateer, C. A. (2001). Attention Process Training Test. Wake Forest, NC: Lash.

Sohlberg, M. M., \& Mateer, C. A. (2009). Reabilitação cognitiva Uma abordagem neuropsicológica. São Paulo, SP: Santos.

Tamm, L., Epstein, J. N., Peugh, J. L., Nakonezny, P. A., \& Hughes, C. A. (2012). Preliminary data suggesting the efficacy of attention training for school-aged children with ADHD. Developmental Cognitive Neuroscience, 4, 16-28. doi:10.1016/j.den.2012.11.004

Tamm, L., Hughes, C., Ames, L., Pickering, J., Silver, C., Stavinoha, P., ...Emslie, G. J. (2010). Attention training for school-aged children with ADHD: Results of an open trial. Journal of Attention Disorders, 14(1), 86-94. doi:10.1177/1087054709347446

Thomson, J., Kerns, K., Seidenstrang, L., Sohlberg, M. M., \& Mateer, C. A. (2005). Pay Attention! A children's attention process training program. Wake Forest, NC: Lash.

Van de Vijver, F. J. R., \& Hambleton, R. K. (1996). Translating tests: Some practical guidelines. European Psychologist, 1, 89-99. doi:10.1027/1016-9040.1.2.89
Recebido: 12/04/2013

I revisão: 09/09/2013

Aceite final: 08/10/2013 